\title{
Bypassing PACU: a cost effective measure
}

Discharge criteria for the twenty-first century can SAFE (Short-acting, fast emergence) anaesthetics make a difference? Do all patients really need to go to PACU?

Until recently, recovery from general anaesthesia in the United States was deemed acceptable if the patient could be safely transferred from the recovery room to the general surgical ward of the hospital. With the luxury of unlimited reimbursement from third party payers, further recuperation, even from a simple 10 min surgical procedure, might take hours, even days. Today, as government, industry, and third party payers continue to ratchet the practice of medicine in America, the luxury of a prolonged recovery appears to be extinct.

With a continued trend to do more complex ambulatory surgery, which often requires a longer duration of anaesthesia, the assessment of recovery from anaesthesia becomes an increasingly critical clinical issue. The recovery room is no longer the "cash cow" it was in a fee-for-service system. In fact, the pendulum has swung to the opposite extreme. In this environment of managed care and capitated payments, providing an intensive care setting in a PACU for healthy patients who may actually be fully "recovered" when they are admitted to the PACU is likely costing the facility thousands, tens of thousands, or even hundreds of thousands of dollars annually. As the subspecialty of ambulatory anaesthesia continues to grow, it becomes increasingly important for the anaesthetist to assess accurately the earliest possible time when patients can be safely sent home after surgery, e.g., immediately at the conclusion of the surgical procedure while the patient is still in the operating room. A review of current standards of care established by the JCAHO and the ASA both appear to permit this practice. Indeed, the current standards for post-anaesthesia care as approved by the American Society of Anesthesiologists states:

The post-anesthesia care unit (PACU) or an area which provides equivalent post-anesthesia care shall be available to receive patients after surgery and anesthesia. All patients who receipe anesthesia shall be admitted to the PACU except by specific order of the anesthesiologist responsible for the patient's care." (emphasis to the word except as specified in the ASA Standards for Post-Anesthesia Care document).

Important lessons from the SAFE (Short-acting, fast emergence) study

The SAFE Study was designed to evaluate the rapid recovery of patients undergoing ambulatory surgery using short-acting, fast emergence anaesthetic agents; to determine if policies and procedures could be developed that would allow patients to bypass first stage post-anaesthesia care units (PACU) safely; and to see whether such changes in the recovery paradigm would result in financial savings for the surgical centre. Five community-based facilities (hospitals or surgery centres) participated in this prospective observational study. While in the operating room at the end of the surgical procedure, anaesthetists were instructed to assess all ambulatory surgical patients for recovery with standard discharge criteria typically used at the end of a PACU stay (see Table below). If the patients met the discharge criteria, they were transferred from the OR directly to the less labour intensive second stage recovery unit (SSR). Financial data were provided from all five sites

\section{TABLE Discharge criteria}

- Awake, alert, oriented, responsive (or return to baseline)

- Minimal pain

- No active bleeding

- Vital signs stable (not likely to require pharmacological intervention)

- Minimal nausea

- No vomiting

- If nondepolarising neuromuscular blocking agent used, patient can perform five-second head lift

- Oxygen saturation of $94 \%$ on room air (three minutes or longer) or return of oxygen saturation to baseline or higher

In order to be eligible to bypass Phase I recovery (PACU), the patient must meet all of the above criteria and, in the judgment of the anaesthetist, be capable of transfer to the step-down unit, with appropriate care and facility for patient management at that location.

From the Department of Anesthesia and Critical Care, University of Chicago, 5841 S Maryland, MC 4028, Chicago IL 60637 USA 
detailing all costs associated with the recovery process. Clinical data on every elective ASA 1, 2 and 3 ambulatory surgical patient were collected over three months. During month one, data collected established a baseline of case mix, timestamps, adverse events, bypass rates, and financial data at each site. During month two, an educational intervention was provided on a multi-disciplinary basis to all units in the surgical centre discussing the implications of the bypass paradigm. After implementation of the paradigm (month three) weekly feedback reports were provided to the site featuring the key outcomes of the study, and these reports were distributed to the health care providers.

Nearly 5,000 patients were entered into the study. While baseline bypass rates following general anaesthesia ranged from 0 to $2 \%$, bypass rates for patients who received general anaesthesia in the month following the educational intervention ranged from 14 to $42 \%$. At all five sites, patients who bypassed the PACU had considerably reduced total recovery time compared with patients who did not bypass. There were no differences in other parameters of patient outcome. Annualised savings ranged from $\$ 50,000$ to $\$ 160,000$ per site.

The results of this study suggest that important cost savings can be achieved without compromising patient safety after implementing a bypass paradigm. A team approach to an educational intervention and paradigm implementation, along with objective, data driven, ongoing feedback to clinicians and administrators on patient and process outcomes were key factors in achieving the financial success experienced at each site. 


\section{Éviter la salle de réveil: une mesure rentable}

Critères de congé pour le $21^{\mathrm{e}}$ siècle - Les agents anesthésiques SAFE (Short Acting, Fast Emergence) peuvent-ils faire la différence? Est-ce que tous les patients doivent passer par la salle de réveil ? Jusqu'à récemment, la récupération de l'anesthésie générale aux États-Unis était considérée acceptable si le patient pouvait être transféré sécuritairement de la salle de réveil à l'unité de soins chirurgicaux de l'hôpital. Avec le luxe d'un remboursement sans limites par un tiers payeur, la suite de la récupération, même suite à une procédure simple de 10 minutes, pouvait prendre des heures et même des jours. Aujourd'hui, au moment où le gouvernement, l'industrie et les tiers payeurs continuent à contraindre la pratique de la médecine en Amérique, le luxe d'une récupération prolongée semble disparu.

Avec la tendance continue à faire des procédures plus complexes en chirurgie ambulatoire, requérant des anesthésies de plus longue durée, l'évaluation du réveil de l'anesthésie devient une question clinique de plus en plus critique. La salle de réveil n'est plus la vache à lait qu'elle était dans le système du paiement à l'acte. En fait, le pendule a oscillé à l'autre extrême. Dans cet environnement de "managed care» et de capitation, le fait de fournir un environnement de soins intensifs dans une salle de réveil pour des patients en santé qui peuvent être en fait complètement éveillés à leur admission dans cette salle de réveil coûte probablement à l'unité chirurgicale des milliers, des dizaines de milliers sinon des centaines de milliers de dollars annuellement. Avec le développement de l'anesthésie ambulatoire, il devient de plus en plus important pour l'anesthésiste d'évaluer précisément le moment le plus précoce où le patient peut sécuritairement être renvoyé à la maison après la chirurgie, par exemple immédiatement à la fin de la procédure chirurgicale quand le patient est encore en salle d'opération. Une revue des normes de soins actuelles telles qu'établies par JCAHO et l'ASA semble permettre cette pratique. En effet, les normes actuelles de soins post-anesthésiques telles qu'approuvées par l'ASA disent : "Une salle de réveil ou un endroit permettant
des soins post-anesthésiques équivalents doit être
disponible pour recevoir les patients après l'anesthésie
et la chirurgie. Tous les patients recevant une

\begin{abstract}
anesthésie doivent être admis à la salle de réveil sauf sur ordonnance spécifique de l'anesthésiologiste responsable des soins du patient". (L'emphase est mise sur le mot sauf dans le document "ASA standards for Post Anesthesia Carem.)
\end{abstract}

Leçons importantes tirées de l'étude SAFE (Short-acting, fast emergence)

L'étude SAFE a été mise sur pied pour évaluer le réveil rapide de patients, en chirurgie ambulatoire, utilisant les agents anesthésiques à courte durée d'action et à émergence rapide et pour déterminer si des règles et procédures pouvaient être produites pour permettre aux patients d'être exemptés de façon sécuritaire d'un séjour dans une salle de réveil conventionnelle (niveau I), et si tous ces changements dans le paradigme du réveil entraîneraient des économies financières pour les centres de chirurgie. Cinq centres non universitaires (hôpitaux ou centres de chirurgie) ont pris part à cette étude prospective et basée sur l'observation. Alors qu'ils étaient encore en salle, à la fin de l'opération, on demanda aux anesthésistes d'évaluer tous leurs patients de chirurgie ambulatoire quant à leur état d'éveil en utilisant les critères typiques utilisés lors du congé de la salle de réveil (Tableau). Si les patients rencontraient ces critères de sortie, ils étaient trans-

\section{TABLEAU Critères de sortie.}

- Éveillé, alerte, orienté, capable de répondre (ou état avant anesthésie).

- Douleur minime.

- Aucun saignement actif.

- Signes vitaux stables (aucune intervention pharmacologique requise).

- Nausées minimes.

- Aucun vomissement.

- Après usage de bloqueurs neuromusculaires, le patient est en mesure de tenir sa tête levée pour 5 secondes.

- Saturation en oxygène de $94 \%$ à l'air libre (pour 3 minutes ou plus) ou le retour de la saturation à la valcur préanesthésique ou mieux.

Pour pouvoir être éligible à court-circuiter la salle de réveil de $1^{\text {er }}$ niveau, le patient doit rencontrer tous ces critères et, selon le jugement de l'anesthésiste, être capable d'être transféré à une unité de second niveau offrant des soins appropriés et une capacité de prise en charge du patient. 
férés directement de la salle d'opération (SOP) à une unité de réveil de second niveau (SSR) nécessitant moins de personnel. Des données financières ont été recueillies dans les cinq centres évaluant tous les coûts associés au processus de réveil. Des données cliniques ont été recueillies durant 3 mois concernant chaque patient en chirurgie ambulatoire élective de classe I, 2 ou 3 de l'ASA. Durant le premier mois, les données réunies ont permis d'établir une ligne de base concernant la variété des cas, les durées, les complications, les taux de court-circuitage (bypass) de la salle de réveil ainsi que les données financières, et ce pour chaque centre. Durant le second mois, une intervention à visée éducative a été réalisée sur base multidisciplinaire auprès de toutes les unités des centres de chirurgie et les implications du paradigme court-circuitage ont été discutées. Après la mis en oeuvre du paradigme, c'està-dire au troisième mois, des rapports hebdomadaires de rétroaction étaient fournis au site ayant atteint les objectifs clés de l'étude et ces rapports étaient distribués aux professionnels.

Près de 5000 patients ont fait partie de cette étude. Alors que le taux de base de court-circuitage à la suite d'une anesthésie générale s'établissait à $0-2 \%$, ce taux grimpa à $14-42 \%$ dans le mois suivant l'intervention éducative. Sur les cinq sites, les patients qui courtcircuitaient la salle de réveil avaient un temps de réveil total considérablement moindre que ceux qui y étaient admis. Il n'y avait pas de différence quant aux autres paramètres de devenir des patients. Les économies variaient de $\$ 50,000$ à 160,000 par année et par site.

Les résultats de cette étude suggèrent que des économies importantes peuvent être réalisées sans compromettre la sécurité des patients après avoir mis sur pied un paradigme de court-circuitage de la salle de réveil. Les facteurs-clés du succès financier réussi à chaque site sont: unc approche d'équipe pour une intervention éducative et pour l'implantation du paradigme couplée à une rétroaction continuelle objective, basée sur des données, à l'intention des cliniciens et des administrateurs et portant sur les résultats tant pour les patients que pour le processus lui-même. 